\title{
An Agile Innovation Framework Supported through Business Incubators
}

\author{
Ozgul Unal, Hamideh Afsarmanesh, and Samuil Angelov \\ Informatics Institue, University of Amsterdam, \\ Science Park 1071098 XG Amsterdam, The Netnerlands \\ \{O.Unal-Karakas, H.Afsarmanesh, S.Angelov\}@uva.nl
}

\begin{abstract}
Entrepreneurs and SMEs that develop new ideas are the main sources of innovation in products and services in the society. However, due to the lack of required resources and know-how innovation ideas may not develop into full-fledged results. Business incubators can play an important role here. They can provide innovators with the required enabling environment, guidelines, and tools supporting the innovation process. This paper proposes an enabling agile innovation framework, which is based on using VBE and VO paradigms. The paper first defines the innovation process, and then addresses a set of tools and mechanisms that need to be utilized to facilitate the innovation process. An example case in intelligent buildings is also provided.
\end{abstract}

Keywords: Business incubators supported innovation, innovation framework, innovation process, innovation in VBEs and VOs.

\section{Introduction}

Small and medium enterprises (SMEs) increasingly feel the pressure of global competition. To survive through the current market conditions, they need to respond to customers' demands much more efficiently and effectively than ever before. One approach to achieve this goal is through continuous improvement, and another through innovation in products and services. As defined in [1], "An innovation is something original, new, and important - in whatever field - that breaks in to (or obtains a foothold in) a market or society".

In spite of the large amounts of effort and interest presented in the literature related to this area, it is reported that most innovation projects fail. The main reasons, a number of which are also expressed in [2,3] include the following:

- Innovating organization's goals are ineffectively deployed into actions.

- Organization does not have an enabling innovation management framework.

- Financial resources are not sufficient for developing the prototype of the idea.

- Planning, design, and development of the innovations are poor.

- Access to the required information and knowledge is poor.

- ICT support is not efficiently and/or sufficiently used.

- Inter- and intra-organizational collaboration is not sufficiently exploited. 
- The processes of finishing the innovation project and introducing its results to customers are slow.

- There is no mechanism to monitor and assess the whole innovation process.

A number of research efforts have focused on overcoming some of the listed problems, although with some limitations, as discussed in Section 2. But the biggest and the most common problem for innovation as reported by entrepreneurs and SMEs alike is the lack of necessary resources and know-how to properly develop their ideas. Business incubators possess the required expertise and therefore can support in this process. However, an innovation enabling framework which can be provided through the incubators to the entrepreneurs and SMEs is still missing.

Taking into consideration the common problems and challenges in innovation efforts, and focusing on aspects not yet addressed by the literature on related research, this paper proposes an enabling agile innovation framework. This framework is based on the concepts introduced in Virtual Organization Breeding Environment (VBE) [4, 5] and in Virtual Organizations (VOs) [6] paradigms, and introduces an innovation process as well as a set of assisting tools and methodologies to be used during the execution of this process. The business incubators domain is considered as the main domain for applying our innovation framework.

The remaining of this paper is structured as follows. Section 2 provides information about related work. Section 3 describes our framework and its main components. Section 4 provides an example, to show how an innovative idea gets processed by the steps of our proposed framework and the main outputs of the innovation process. Finally, Section 5 concludes the paper.

\section{$2 \quad$ Related Work and Open Issues}

This section provides the main examples of related research in this area. They are grouped here into four categories, based on the main focus area addressed by them, including: 1) open innovation, 2) collaborative innovation and innovation networks, 3 ) the role of $\mathrm{VO} / \mathrm{VBE}$, and 4) the role of ICT.

The first group of related work focuses on open innovation. For example, [8] and [9] focus on open innovation in SMEs. Open innovation is primarily introduced by Henry Chesbrough [7], who defines it as follows:

"Open innovation is a paradigm that assumes that firms can and should use external ideas as well as internal ideas, and internal and external paths to market, as the firms look to advance their technology."

The second group of related work focuses on collaborative innovation. The idea of innovation networks and collaborative innovation networks has become popular during the recent years and thus become the focus of research efforts, such as [11] and [12]. A Collaborative Innovation Network (CoIN) is defined by its original proposer, Peter Gloor [10], as follows:

"A cyberteam of self-motivated people with a collective vision, enabled by the Web to collaborate in achieving a common goal by sharing ideas, information, and work."

The third group of related work focuses on the role of VBE and VO paradigms in innovation. The VBE and VO are two main types of Collaborative Networks (CNs) that have drawn considerable amount of attention. A VBE [4, 5] represents a long 
term alliance established among large number of organizations. VBE provides the necessary conditions and mechanisms to its members to support them with their need to potentially configure goal-oriented Virtual Organizations (VOs) [6]. A VO is established among a number of VBE members, when an emerged opportunity can be supported through their collaboration and joining their forces and resources.

VBE and VO have been considered by only a few efforts in the context of innovation. In [13], VO paradigm is used in the formation of virtual teams. In [14], the authors address the need for a methodological support on how to structure and conduct innovation processes in VOs. They propose VBE as the facilitator for an agile strategic innovation management for VOs.

The fourth group of related work comprises the efforts focusing on the role of ICT in innovation. In addition to emphasizing the importance of ICT, proposing ICTbased innovation support infrastructures has been the focus of a number related research efforts, such as [15] and [2].

Although there are large numbers of innovation related research efforts, most of them focus only on a single aspect related to innovation, such as: open innovation, or collaborative innovation network. Furthermore, although there are a few efforts addressing VO/VBE paradigms as key enablers for agile innovation process, the details of how these paradigms can be used for innovation is not yet investigated. A comprehensive approach for innovation is needed that encompasses a framework, based on innovation related paradigms, and providing both well-defined innovation process and guidelines on which tools and mechanisms to use at each step.

\section{Proposed Agile Innovation Framework}

Considering the reasons for the failure of most innovation projects and the limitations of other innovation related efforts in the literature, we propose a framework that combines technologies and conceptual paradigms to provide a comprehensive solution. This framework consists of two main building blocks: 1) innovation process definition, and 2) a set of tools/mechanisms to facilitate the execution of the process. More details are provided in Sections 3.1 and 3.2. The main underlying paradigms of this framework are the VBE and VO, which support the collaborative and open innovation paradigms. The main advantages of VBEs/VOs for the success of the innovation efforts are as follow:

- As [12] states, VBE can support the exploitation of local competencies and resources by an agile and fast configuration of the most adequate set of partners for each innovation project. This also enables producing economically viable solutions for innovative ideas.

- Trust management mechanisms are provided in VBEs, which enhances the cooperation/collaboration among member organizations, once within the VOs.

- VBE supports well defined roles and policies. Furthermore, mechanisms and guidelines for $\mathrm{VO}$ creation and collaboration are well defined; these act as agility enablers, and can be applied to convince organizations to collaborate.

- Continuous monitoring of VBE members' qualifications helps with both upholding competency level and gap identification to take corrective actions. 
- Issues such as sharing only the necessary resources with other appropriate partners and IPR are well established in VBEs and support member autonomy.

- VBEs provide an effective common ICT infrastructure, which is indispensable for successful innovation projects, supporting needed agility in VO formation.

Considering these advantages, the framework proposed in this paper is based on the VBEs and VOs as key enablers. Furthermore, we have selected businessincubator-supported innovation as the main application domain. There are many entrepreneurs and SMEs that have innovative ideas, but do not have the necessary experience and resources to develop them. Business incubators provide the necessary means to support successful development of ideas into products. As part of this support, it is also necessary to provide innovators with a framework that consists of a guideline and a set of suggested tools/mechanisms to be used during the innovation process, which makes business incubator supported innovation a good-fit application domain for our framework.

Since the framework is based on the VBE, it is assumed that the incubators are also members of the VBE. Furthermore, when signing a contract with business incubators, the innovators (being entrepreneurs or SMEs) also become a member of this VBE. Many other production and delivery companies and financial organizations constitute the other members of the VBE. Innovation VOs interact with customers throughout the innovation process in order to involve them at all phases.

\subsection{Innovation Process}

To improve understandability of innovation process, first a number of terms used in this process are defined. A Goal is defined by an innovator to represent the main aim of an innovative idea. The innovator may divide/decompose a goal into Subgoals, in order to make it easier to achieve the goal. A Subproduct is a smaller unit of a product. A Competency for an organization corresponds to one capability or capacity.

The innovation process proposed and described below is rooted in and represents an enhancement of the results reported in multiple resources, such as the Stage-Gate Model [16]. This process specifies the actions to be taken throughout the innovation process, and is therefore of great value to be provided by business incubators to inexperienced entrepreneurs and SMEs. As we assume that ideas are already defined, some steps of the pre-development activities of New Product Development process (e.g. opportunity identification, opportunity analysis, idea genesis, and idea selection [17]), are not included in this process. Innovation process, provided by business incubators to innovators as an explanation of what to do at each step, as depicted in Figure 1, includes the following sixteen steps:

1. Subgoals definition: Innovators decompose the goal into subgoals.

2. Market research: Based on the subgoals, market research is carried out in order to identify the needs and constraints.

3. Requirements definition: Requirements for each subgoal are defined.

4. Draft solutions design: Draft(s) of possible solution(s), which meet the defined requirements, are generated at this step e.g. by means of brainstorming. Draft list of required subproducts might be identified at this step. 


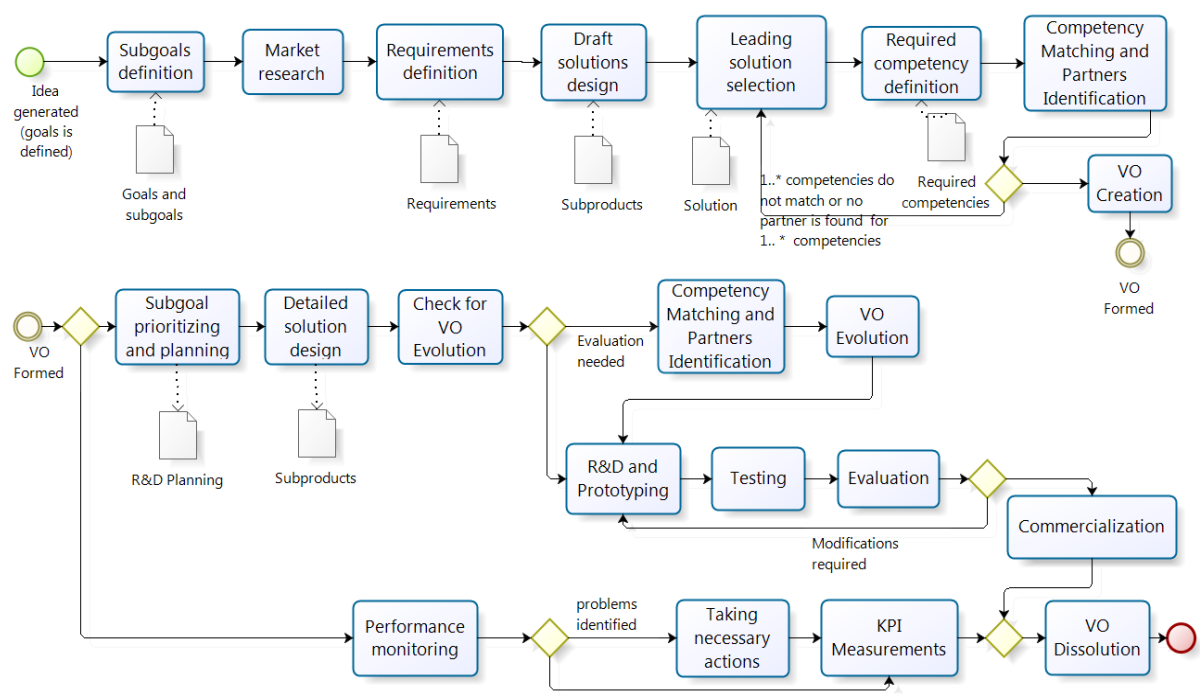

Fig. 1. Innovation Process

5. Leading solution selection: Considering the market needs and resource requirements (e.g. finances, time, people, materials, etc.), a number of metrics is defined for selecting the most suitable solution. Then, solutions are ranked and the first ranked solution is selected.

6. Required competencies definition: For each subgoal, competencies required for accomplishing the selected solution are identified. The terminology used by innovators might be syntactically and/or semantically different from the competencies specified in the VBE.

7. Competency matching \& potential partners identification: Required competencies are automatically matched against the competencies of the VBE members in order to identify the potential partners in the innovation VO.

8. Innovation VO creation: The trustworthiness of the potential partners is evaluated to shortlist them. Then, a negotiation round takes place to identify the most suitable partners. The VO plan is detailed out and a contract is signed by each of the selected partners, which essentially marks the official launch of the VO.

9. Subgoal prioritizing and planning: It is identified by the VO members (including innovators) in which order the subgoals shall be achieved. Then, a detailed plan for the whole development process is made.

10. Detailed solution design: For each subgoal, a detailed solution is designed and subproduct(s) to be utilized or developed are identified.

11. Checking if VO's evolution is necessary: If new competencies are required according to the detailed solution and none of the VO partners possess this competency, than VO enters into an evolution phase.

12. $R \& D$ and Prototyping: The needed research and development activities are carried out and a prototype (e.g. physical) is produced at this step.

13. Testing: The developed prototype is tested to make sure that it works properly.

14. Evaluation: Prototype is evaluated by VBE members, through discussion forums. 
15. Commercialization: Production is launched and final product is distributed to the market. Advertisements are also placed at this step.

16. VO Dissolution: Since the goal of the VO is reached, it is dissolved.

When a VO is created at Step 8, then the VO Operation phase starts, which needs to be continuously monitored. Therefore, a Monitoring activity runs in parallel to steps 9-15.

After the dissolution of the innovation VO, a number of activities need to be continuously carried out by the innovators initiating the process, such as evaluating the product, monitoring the market, and analyzing internal/external value and cost. Furthermore, although the VO is dissolved after the initial production of the idea, other types of VOs might need to be created later on, for example for mass production.

In addition to the main innovation process explained above, there are also some auxiliary processes (e.g. managing trust related data, managing member data, and managing competence data [18]) of which their details are outside the scope of this paper.

\subsection{Supporting Tools/Mechanisms}

In order to facilitate the execution of the innovation process, automated tools and mechanisms are needed. For innovation purposes, two main types of systems can be considered as part of the innovation framework.

One type of assisting system can address the management of novel ideas. Such a system may consist of tools that can for instance be used for idea mining and/or determining the idea uniqueness. Since we assume that innovators already have unique novel ideas, the idea management falls outside the current scope of our work.

Another type of system can address Creation/operation of innovation VOs inside the VBEs. As shown in Figure 2, our designed VO Creation/Operation System consists of:

1) Competence Matching: At Step-6 of the innovation process, innovators identify the competencies that are required to successfully achieve the subgoals. Since these competencies are specified in the terminology (or keywords) of innovators, at Step-7 they need to be matched against the competencies defined in the VBE, to find most suitable partners. $\mathrm{P}$ erforming this match manually by human is time consuming and error prone. The Competence Matching component can achieve this automatically by exploiting a number of syntactic and semantic matching algorithms and thus facilitates rapid formation of the innovation VO. Due to potential syntactic/semantic conflicts between these two specifications of competencies, user intervention might be required after the matching process has produced its results.

2) Trustworthiness Evaluation: Competence matching results in a list of potential VO members, which then gets refined based on their trustworthiness. The component for evaluation of organization trustworthiness can be built on top of the TrustMan system [19], developed previously.

3) Negotiation Support: After the potential most-fit members of the innovation VO have been identified, a negotiation round takes place. First, the negotiation objects, which require agreements, are defined. Then potential members discuss 
about these objects through collaboration tools such as chat and forum. The results are reflected in the VO contracts. This component utilizes the Negotiation Wizard developed within the ECOLEAD project [20].

4) Performance Evaluation: During the operation phase of the life cycle of the innovation VO, performance of its members needs to be continuously monitored and measured. This is important for the success of the innovation VO. For this purpose, a set of Key Performance Indicators (KPIs) need to be defined and the performance of each member needs to be measured against these indicators, which would be addressed in future work.

The innovation VO Creation/Operation System is further extended with a number of VBE-related subsystems. The subsystems (indicated as related subsystems in Figure 2), are developed in two EU-funded projects, namely ECOLEAD [20] and GloNet [4]. Since these subsystems are auxiliary and considering space limitations, no details are provided on them, but the related information can be found in [18].

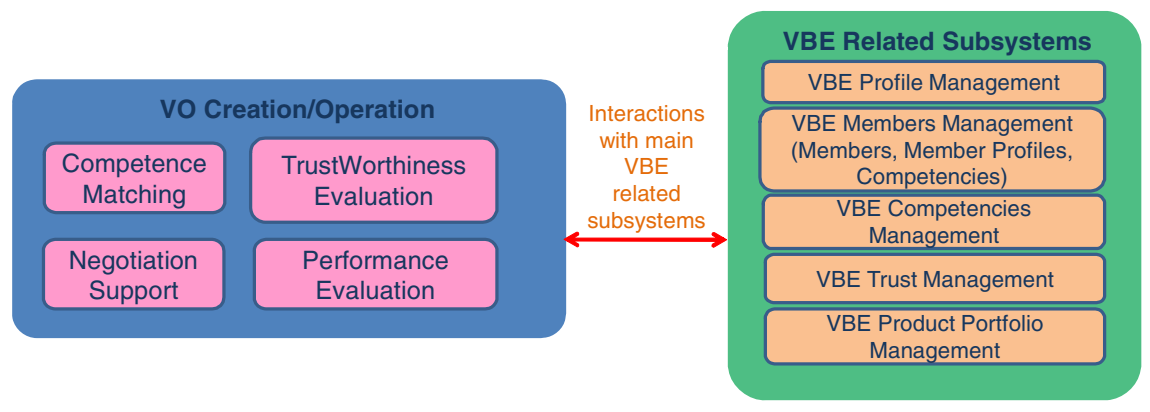

Fig. 2. Components of the VO Creation/Operation System

\section{$4 \quad$ An Example Case (Intelligent Buildings)}

This section provides an example from the intelligent buildings domain (based on [21]) to illustrate the main outputs of the innovation process. For space reasons, only the main steps of the innovation process and a partial sample list of competencies are addressed here.

Imagine the following being considered by some innovators. In buildings, each occupant has his/her own preferences related to the temperature and light. An advanced service supported in an intelligent building system, which consists of sensors (e.g. temperature detectors) and devices (e.g. heaters), may learn these preferences over time. When a person enters a room in a building, it should be detected who entered the room, so that the temperature and the light parameters can be automatically adapted according to his/her preferences. In simpler cases, such as in the case of an environmental change (e.g. the weather gets dark) the conditions should be adapted accordingly. For an intelligent building, it is also important to react rapidly and correctly in an emergency situation. For example, when a fire starts in a room, this needs to be immediately detected and shared with other parts of the system in other rooms, which requires that all sensors and devices are connected through a 
network. Similarly, if the weather is dark during a fire situation, the light level needs to be increased for faster evacuation.

Based on the scenario explained above, the following main goal corresponding to the innovative idea can be defined by the innovators:

Goal: The rooms of a building should adapt both to the needs of its occupants and the circumstances, including adapting the lighting and temperature, to support the needs of the person currently in the room as well as to better respond to an emergency situation, such as a fire.

Then, the following subgoals can be defined by innovators:

Subgoal 1: Ensure that a person entering a room can be identified.

Subgoal 2: Ensure that temperature and lighting preferences/needs of persons living in the building are learned over time.

Subgoal 3: Ensure that a room can automatically adapt to its current occupant's light and heat parameters, so that the occupant is satisfied in the environment.

Subgoal 4: Ensure that a fire situation can be immediately diagnosed and reacted upon, by providing a more suitable lighting.

After subgoals have been defined, a market research is carried out to identify the functional and non-functional requirements for the intelligent building systems (not addressed with more details due to space limitations). Then, draft solutions for the systems are designed to meet the requirements. Different solutions may result in different subproducts needed for its realization. For example, one solution may need sub-products including: Agents, Sensors (temperature, light level, movement, smoke), Effectors (heating, lighting, doors, windows, alarm), Network connection, and Software (to gather information from sensors, to generate a response).

After a solution has been selected, required competencies are identified by innovators in order to achieve the corresponding subgoal. An example list of competencies is: 1) Software design, 2) Development, 3) Testing, 4) Mobile robot control techniques, 5) Sensor manufacturing, 6) Sensor development, 7) Networks supporting.

Table 1. Competency Matching and Potential VO Members Identification

\begin{tabular}{|c|c|c|}
\hline $\begin{array}{c}\text { Results of Competency Matching } \\
\text { (Defined competency;VBE Competency) }\end{array}$ & $\begin{array}{l}\text { Which Organizations have } \\
\text { which VBE Competencies }\end{array}$ & $\begin{array}{l}\text { Potential } \\
\text { VO } \\
\text { Members }\end{array}$ \\
\hline $\begin{array}{l}\text { (Software design;Software analysis and design) } \\
\text { (Development;Software development) } \\
\text { (Testing;Software testing) } \\
\text { (Mobile robot control techniques;Mobile robots) } \\
\text { (Sensor manufacturing;Sensor manufacturing) } \\
\text { (Sensor development;Sensors development) } \\
\text { (Networks supporting;Computer networks) }\end{array}$ & $\begin{array}{l}\text { Software analysis and } \\
\text { design: A, B } \\
\text { Software development: A, B, } \\
\text { C } \\
\text { Software testing: A, B, C } \\
\text { Mobile robots: D } \\
\text { Sensors manufacturing: E, F } \\
\text { Sensor development: F, G } \\
\text { Computer networks: H }\end{array}$ & $\begin{array}{l}\text { A, B, C, } \\
D, E, F, \\
G, H\end{array}$ \\
\hline
\end{tabular}


Then, the competency matching software component helps to automatically match the innovators' specified competencies to the VBE competencies. Based on the match results, a preliminary list of VO members is identified. In relation to this example, we assume that VBE competencies are the ones shown after the ";" symbol, in the first column of Table 1. We also assume that organizations $A, B, C, D, E, F, G$, and $H$ have those VBE Competencies that are shown in the second column of the table.

Due to space reasons, we do not discuss the remaining steps of the innovation process, as these are related to the operation of the VO.

\section{Conclusion and Future Work}

An agile innovation framework is proposed in this paper. This framework is especially meant for innovators supported by business incubators and aims to guide the innovators through the innovation process on what to do and which tools / mechanisms to use at each step. Considering the many benefits that VBE and VO provide for the innovation process, they form the base of the framework.

As part of the future work, the plan is to implement the Competence Matching Component and to make the necessary adaptions to the Trustworthiness Evaluation and Negotiation Support Components developed in the ECOLEAD Project. Then, the Performance Evaluation Component is planned to be implemented. For that purpose, first the KPIs will be defined, which will then be used by the Performance Evaluation Component to compute the innovativeness of the VO members. The related data stored during the life cycle of a VO can then be used when developing the future VOs, in the same way that trust data are used.

Acknowledgments. The work on this paper is partially supported by the FP7 project GLONET.

\section{References}

1. Frankelius, P.: Questioning two myths in innovation literature. Journal of High Technology Management Research 20(1), 40-51 (2009)

2. Dooley, L., O'Sullivan, D.: Developing a software infrastructure to support systemic innovation through effective management. Technovation 23(8), 689-704 (2003)

3. Pyka, A., Küppers, G.: Innovation Networks: Theory and Practice. Edward Elgar, Cheltenham (2002)

4. Camarinha-Matos, L.M., Afsarmanesh, H., Koelmel, B.: Collaborative Networks in Support of Service-Enhanced Products. In: Camarinha-Matos, L.M., Pereira-Klen, A., Afsarmanesh, H. (eds.) PRO-VE 2011. IFIP AICT, vol. 362, pp. 95-104. Springer, Heidelberg (2011)

5. Afsarmanesh, H., Camarinha-Matos, L.M., Msanjila, S.S.: On management of 2nd generation Virtual Organizations Breeding Environments. Annual Reviews in Control 33(2), 209-219 (2009)

6. Camarinha-Matos, L.M., Afsarmanesh, H.: A framework for virtual organization creation in a breeding environment. Annual Reviews in Control 31(1), 119-135 (2007) 
7. Chesbrough, H.W.: Open Innovation: The new imperative for creating and profiting from technology. Harvard Business School Press, Boston (2003)

8. Lee, S., Park, G., Yoon, B., Park, J.: Open innovation in SMEs-An intermediated network model. Research Policy 39(2), 290-300 (2010)

9. Van de Vrande, V., De Jong, J.P.J., Vanhaverbeke, W., Rochemont, M.: De: Open innovation in SMEs: Trends, motives and management challenges. Technovation 29(6-7), 423-437 (2009)

10. Gloor, P.: Swarm Creativity: Competitive Advantage Through Collaborative Innovation Networks. Oxford University Press, Oxford (2006)

11. Johnsen, T., Ford, D.: Managing collaborative innovation in complex networks: Findings from exploratory interviews. In: 16th Annual IMP Conference, Bath, UK (2000)

12. Berasategi, L., Arana, J., Castellano, E.: A comprehensive framework for collaborative networked innovation. Production Planning \& Control: The Management of Operations 22(5-6), 581-594 (2011)

13. Wi, H., Oh, S., Jung, M.: Virtual organization for open innovation: Semantic web based inter-organizational team formation. Expert Syst. Appl. 38(7), 8466-8476 (2011)

14. Eschenbächer, J., Hahn, A.: Strategies for Distributed Innovation management in virtual organisations In: Thoben, K.-D., Pawar, K.S., Weber, F. (eds.): Adaptive Engineering for Sustainable Value Creation, Proceedings of the 10th International Conference of Concurrent Enterprising, Sevilla, Spain, pp. 499-507 (2004)

15. Scupola, A., Tuunainen, V.: Open innovation and role of ICT in business-to-business services: Empirical Evidence from Facility Management Services. In: SIGSVC Workshop. Sprouts: Working Papers on Information Systems, vol. 11(158) (2011)

16. Cooper, R.G.: Winning at New Products. Addison Wesley Publishing Company (1986)

17. Koen, P., Ajamian, G., Burkart, R., Clamen, A.: Providing clarity and a common language to the "fuzzy front end". Research Technology Management 44(2), 46-55 (2001)

18. Camarinha-Matos, L.M., Macedo, P., Ferrada, F., Oliveira, A.I., Afsarmanesh, H., Unal, O.: Specification of support services for management of long-term base network. EU Project (285273) - Deliverable 5.11, Global enterprise network focucing on customercentric collaboration (2013)

19. Msanjila, S.S., Afsarmanesh, H.: On development of TrustMan system assisting configuration of temporary consortiums. International Journal of Production Research; Special Issue: Virtual Enterprises - Methods and Approaches for Coalition Formation 47(17), 4757-4790 (2009)

20. Camarinha-Matos, L.M., Silveri, I., Afsarmanesh, H., Oliveira, A.I.: Towards a framework for creation of dynamic virtual organizations. In: Camarinha-Matos, L.M., Afsarmanesh, H., Ortiz, A. (eds.) Collaborative Networks and their Breeding Environments. IFIP, vol. 186, pp. 69-80. Springer, Boston (2005)

21. Sharples, S., Callaghan, V., Clarke, G.: A Multi-Agent Architecture for Intelligent Building Sensing and Control. International Sensor Review Journal 19(2), 135-140 (1999) 